\title{
Ultrasound guided fine needle aspiration cytology of intra-abdominal masses in a tertiary center of Bihar, India
}

\author{
Namita KUMARI', Amar RANJAN*2 \\ ${ }^{1}$ Department of Pathology, Patna Medical College, Patna, Bihar, India \\ ${ }^{2}$ Lab Oncology, DR. BRA Institute-Rotary Cancer Hospital, All India Institute of Medical Sciences, New Delhi, India
}

Introduction: Ultrasonography (USG)-guided fine needle aspiration cytology (FNAC) has become an indispensable component of diagnosis of abdominal masses especially the pancreato-biliary system. It allows the real time visualization of the needle tip as it passes through the tissue planes into the target area. Under USG guidance, needle can access smaller lesions and also lesions in critical anatomical areas.

Methods: The study involved 50 cases with clinically or ultrasonographically diagnosed of abdominal masses conducted in the department of pathology, Patna Medical College, Patna, India.

Results: Among total 50 patients in the study, $33(66 \%)$ were female and $17(34 \%)$ were male. The age ranged from 4 to 75 years. The most common site of FNAC was Gall bladder, being $38 / 50$ (76\%). Among the total 50 cases, 28 (56\%) were malignant, $17(34 \%)$ were benign and $5(10 \%)$ were inconclusive. The most common age group was 51 to 60 years constituting 15/50 (30\%) cases. Out of these 15 cases, 13 were malignant and 2 were benign. The most common malignancy seen, was carcinoma of Gall bladder, predominantly in females living in Gangetic belt (people living near the river Ganga) of Bihar, were the Arsenic content of soil is said to be very high.

Conclusions: USG-guided FNAC procedure involves the presence of both a pathologist and a radiologist, thereby facilitating the exact mass to be hit by the needle for FNAC and appropriate handling of aspirated material for routine and ancillary tests. Real time USG is a simple, cost-effective imaging modality, has an advantage over computed tomography (CT) in guiding non-axial needle trajectories and allowing the procedure to be accomplished more quickly than CT. 